\title{
Low Risk for Marginal Ulcers in Duodenal Switch and Gastric Bypass in a Well-Defined Cohort of 472 Patients
}

\author{
Zakaria Bekhali $^{1,2}$ - Magnus Sundbom ${ }^{1}$
}

Received: 4 May 2020 / Revised: 23 June 2020 / Accepted: 23 June 2020 / Published online: 8 July 2020

(C) The Author(s) 2020

\begin{abstract}
Purpose Marginal ulcer (MU) is well-known complication in bariatric surgery. Several studies are available in Roux-en-Y gastric bypass (RYGBP), while data on the incidence in duodenal switch (DS) is limited. We aimed to compare the incidence of MU between DS and RYGBP in a well-defined cohort and to identify associative factors.

Methods A cohort of 732 patients with BMI $\geq 48$ who had undergone primary DS or RYGBP during 2008-2018 received a questionnaire concerning ulcers, PPI therapy, and smoking habits; hereafter, patient charts were reviewed. Incidence rates (IRs) for MU were calculated in our survey and on previous registered data in the national quality register for bariatric surgery (SOReg). A multivariate regression analysis was performed to identify predictive risk factors for MU.

Results After a mean follow-up of 6.1 years, 472 (64\%) patients responded (47 \pm 11 years old, $65 \%$ women and $42 \%$ DS). Of 41 MUs identified, 23 were endoscopically verified. Gastrointestinal bleeding, abdominal pain, and dysphagia were the most common symptoms. IR for MU was $1.4 \%$ (DS $1.3 \%$ and RYGBP $1.5 \%$ ) per patient-year, compared with $0.9 \%$ according to SOReg-data. Persisting PPI treatment was seen in about three quarter of former MU patients (OR 11.2 [3.6-34.7], $p<0.001)$, but no other associative factors were found.

Conclusion The overall risk for MU was low, about $1 \%$ per patient-year, without difference between DS and RYGBP. Ongoing PPI treatment was frequent in many former MU patients. This study on MU after DS provides reassuring results for future bariatric surgery candidates.
\end{abstract}

Keywords Bariatric surgery $\cdot$ Complications $\cdot$ Duodenal switch $\cdot$ Gastric bypass $\cdot$ Marginal ulcer $\cdot$ Incidence rate $\cdot$ Long term

\section{Introduction}

Severe obesity is associated with increased mortality and morbidity $[1,2]$. Surgical treatment results in sustainable weight loss, reduced comorbidity, and improvement of quality of life $[3,4]$. Among several bariatric procedures, gastric bypass (RYGBP) is well-established worldwide [5] and often considered as gold standard. Duodenal switch (DS) usually

Electronic supplementary material The online version of this article (https://doi.org/10.1007/s11695-020-04822-8) contains supplementary material, which is available to authorized users.

Zakaria Bekhali

zakaria.bekhali@regiongavleborg.se

1 Department of Surgical Sciences, Uppsala University, SE-751 85 Uppsala, Sweden

2 Department of Surgery, Gävle Hospital, Kirurgmottagningen, Gävle sjukhus, SE-801 88 Gävle, Sweden performed on patients with higher BMI $(\mathrm{BMI}>50)$ results in more sustainable weight loss $[6,7]$ but is less frequently performed due to increased technical demands and risk for metabolic complications in the long term [8-10].

Despite the benefits in bariatric and metabolic surgery, operated patients will risk some typical complications such as the development of a marginal ulcer (MU) at, or just distal to, the gastroenteral anastomosis. The pathophysiology is multifactorial, and factors like high acidity, for example, due to large pouch size or gastro-gastric fistula, Helicobacter pylori; local ischemia; and remaining foreign bodies from staple material as well as the used staple technique in itself have been mentioned in many studies [11-18]. Moreover, several patientrelated factors such as smoking, use of corticosteroids and NSAIDs, and various obesity-related comorbidities have also been described $[12,15]$. Abdominal pain is most common symptom of MU, whereas other symptoms include gastrointestinal bleeding or anemia and dysphagia due to stricture [15, 17, 19]. The diagnosis of MU is confirmed with upper 
endoscopy, but a significant proportion of patients with symptoms suggestive of MU are empirically treated by proton pump inhibitors (PPI) [15, 17, 20-22].

The incidence of MU ranges between 0.35 and $16 \%$ in RYGBP $[11,23,24]$ and seems to be low $(0.3 \%)$ in DS [7], although only a limited number of studies exist. In a previous study with wireless $\mathrm{pH}$-metry, we were able to show that the duodenal bulb has a buffering effect, reducing the time with $\mathrm{pH}<4$ from $59 \%$ in the gastric sleeve to $14 \%$ distal to the pylorus. This could theoretically result in lower incidence of MU at the duodeno-ileostomy after DS [25].

In Sweden, bariatric surgery is registered in a national quality register, the Scandinavian Obesity Surgery Registry (SOReg), and since 2007, SOReg contains more than 80,000 patients. Most of them have had a RYGBP (79.2\%), while about $1 \%$ have been operated with DS. Data is collected at baseline, perioperatively, and at follow-up at 6 weeks and 1, 2, 5 , and 10 years. While coverage has been proven high $(97 \%)$ [26], long-term follow-up is not optimal, around $50 \%$ at 5 years.

This retrospective observational cohort study aimed to determine and compare the incidence of MU in DS and RYGBP patients and to find associative factors for development of MU. Furthermore, we have compared our results with registered SOReg-data.

\section{Material and Methods}

Patients having had bariatric surgery at our centers are entered into a local database as well as in SOReg. Postoperatively, patients are followed at our out-patient clinic at 6 weeks and then annually. Data from the perioperative period and the 1and 2-year follow-up are entered in SOReg. In total, 778 patients with $\mathrm{BMI} \geq 48$ having primary DS or RYGBP between January 2008 and September 2018 were identified in local databases. Patients later having had a revisional procedure $(n=27)$ were excluded as were deceased patients $(n=15)$ and those without a known address $(n=4)$ at the time of survey. The 732 selected patients received a paper questionnaire which comprised six questions concerning symptoms, diagnosis, and treatment of postoperative ulcers as well as ongoing treatment with PPI and smoking habits Supplementary Table 1 . In responding patients, we thoroughly reviewed the patient charts, and when we were not clear about any response, we telephoned the patient to ensure clarity. At baseline, comorbid conditions were defined as ongoing treatment of diabetes, hypertension, dyslipidemia, and sleep apnea. Operative time, surgical access, and postoperative complications within 30 days were noted as well as all clinical information on MU during follow-up. Because of the varying duration from surgery, the incidence rate (IR) of MU was determined after calculating years at risk. SOReg-data, containing registrations from routine clinical care in responding patients, was examined similarly.

\section{Statistical Analysis}

Data for age, BMI, and weight are shown as mean \pm standard deviation, unless otherwise noted. Categorical data were analyzed by $\chi 2$ test, while two-tailed Student's $t$ test was used for quantitative data. $p<0.05$ was regarded as statistically significant. For both data sets, the years at risk were calculated subtracting the date of surgery from the time of survey or last clinical follow-up in SOReg, respectively. IR of MU per patient per year was calculated by dividing the total number of MU by number of years at risk. To identify predictors for development of MU, we performed univariate and multivariate analysis including clinically relevant baseline and perioperative data and BMI reduction at 2 years, as well as smoking habits and ongoing PPI treatment. To test equality of two incidence rates for MU between survey and SOReg, we used exact tests due to the small numbers of cases and report mid $p$ values due to the discreteness of the $p$ value. SPSS (IBM, Armonk, NY, USA) was used for all analysis, except the analysis between the two IR which was performed in using STATA (Stata/SE version 15.1 for Windows).

\section{Results}

\section{Included Patients}

In total, 472 patients (64\%) answered the questionnaire, whereof $65 \%$ were women and $42 \%$ had a DS. As demonstrated in Table 1, DS patients had somewhat higher preoperative BMI (56 vs 51) and longer operative time (149 vs $93 \mathrm{~min})$.

\section{Survey}

The cohort had a mean follow-up of $6.1 \pm 2.7$ years. In total, $41(8.6 \%)$ of the patients reported in their questionnaire to have had an MU during the study period, thus resulting in an incidence rate of $1.4 \%$ (DS $1.3 \%$ and RYGBP $1.5 \%$ ), without significant difference between the two procedures ( $p=$ 0.721 ). As demonstrated in Table 2, gastrointestinal bleeding, abdominal pain, and dysphagia were the most common symptoms leading to endoscopy and the subsequent diagnosis of MU. All 23 ulcers were found on the small bowel side of the anastomosis, and in 4 RYPGBs, a stenosis in the gastrojejunostomy was also diagnosed.

Median time from operation to diagnosis of MU was 1.9 (0.1-7.6) years in these 23 patients. The remaining 18 patients had got their diagnosis based on clinical symptoms suggestive of MU. All 41 patients had been treated by PPI. At survey, 
Table 1 Demographics of the included patients and number of marginal ulcers

\begin{tabular}{|c|c|c|c|c|}
\hline & Total & DS & RYGBP & $p$ value \\
\hline Included patients & 472 & $199(42 \%)$ & $273(58 \%)$ & - \\
\hline Women, $n(\%)$ & $308(65 \%)$ & $120(60 \%)$ & $188(69 \%)$ & 0.054 \\
\hline Age at operation (years) & $41.1 \pm 10.7$ & $40.9 \pm 10.3$ & $41.3 \pm 10.9$ & 0.640 \\
\hline Preoperative weight (kg) & $156.8 \pm 23.9$ & $169.6 \pm 25.2$ & $147.5 \pm 17.7$ & $<0.001$ \\
\hline BMI $\left(\mathrm{kg} / \mathrm{m}^{2}\right)$ & $53.7 \pm 5.4$ & $56.8 \pm 6.3$ & $51.4 \pm 3.2$ & $<0.001$ \\
\hline Any comorbidity, $n(\%)$ & $309(65 \%)$ & $140(70 \%)$ & $169(61 \%)$ & 0.057 \\
\hline Operative time (min) & $117 \pm 42$ & $149 \pm 29$ & $93 \pm 35$ & $<0.001$ \\
\hline Surgical access & & & & $<0.001$ \\
\hline Open & $248(52 \%)$ & $130(85 \%)$ & $77(28 \%)$ & \\
\hline Laparoscopy & $215(45 \%)$ & $30(15 \%)$ & $185(68 \%)$ & \\
\hline Converted & $11(2 \%)$ & - & $11(4 \%)$ & \\
\hline Postoperative complication (30 days) & $62(13 \%)$ & $28(15 \%)$ & $34(13 \%)$ & 0.545 \\
\hline \multicolumn{5}{|l|}{ At time of survey } \\
\hline Time from surgery (years) & $6.1 \pm 2,7$ & $6.1 \pm 2.9$ & $6.2 \pm 2,5$ & 0.768 \\
\hline Age (years) & $47.3 \pm 11.1$ & $47 \pm 10.9$ & $47.6 \pm 11.3$ & 0.602 \\
\hline Smoking, $n(\%)$ & $50(11 \%)$ & $21(11 \%)$ & $29(11 \%)$ & 0.986 \\
\hline Ongoing PPI, $n(\%)$ & $123(26 \%)$ & $48(24 \%)$ & $75(27 \%)$ & 0.413 \\
\hline Marginal ulcers in survey, $n(\%)$ & $41(9 \%)$ & $16(8 \%)$ & $25(9 \%)$ & \\
\hline Incidence rate (MU/patient-year) & $1.4 \%$ & $1.3 \%$ & $1.5 \%$ & 0.721 \\
\hline Marginal ulcers in SOReg, $n(\%)$ & $17(2 \%)$ & $8(3 \%)$ & $9(2 \%)$ & \\
\hline Incidence rate (MU/patient-year) & $0.9 \%$ & $1.1 \%$ & $0.7 \%$ & 0.458 \\
\hline Comparison of IR in survey vs SOReg & $p=0.084$ & $p=0.643$ & $p=0.067$ & \\
\hline
\end{tabular}

Data presented as mean (SD) if not otherwise stated there was no significant difference between the two procedures regarding ongoing PPI treatment $(24 \%$ vs $27 \%, p=$ 0.413 ) nor smoking habits ( $11 \%$ for both). The main indications for ongoing PPI treatment were abdominal pain (50\%) and gastroesophageal reflux (24\%).

In both the univariate and multivariate analysis, there was a significant association between a former MU and ongoing PPI treatment (OR 11.2 [3.6-34.7], $p<0.001)$. No other associative factors were found Table 3 .

\section{SOReg-Data}

For SOReg-data, the incidence rate for MU in our DS and RYGBP was 1.1 and $0.7 \%$, respectively, with no significant differences between the two procedures $(p=0.458)$. Compared with the results from the present survey, there was a trend towards a lower overall risk for MU $(0.9 \%$ vs $1.4 \%, p=0.084)$ as well as in RYGBP $(0.7 \%$ vs $1.5 \%, p=$ $0.067)$.

\section{Discussion}

In the present cohort of 472 patients with an initial BMI $>48$, we were able to show a low incidence rate for marginal ulcers in DS and RYGBP, $1.3 \%$ and $1.5 \%$, respectively, during a mean follow-up of 6.1 years. As expected, abdominal pain, gastrointestinal bleeding, and dysphagia were the most common symptoms leading to endoscopy and a diagnosis of an MU. Of the endoscopically verified MU patients, $57 \%$ were diagnosed within the first 2 postoperative years. Persisting PPI treatment was seen in $73 \%$ of former MU patients, but no other associate factor could be determined.

Our survey is one of the few that describes the incidence of MU after DS. We chose to include only patients with BMI $\geq$ $48 \mathrm{~kg} / \mathrm{m}^{2}$, having had primary bariatric surgery without any revisional procedure, to make the two groups as comparable as possible. Because of our clinical patient selection mechanisms, the studied DS patients had somewhat higher BMI, while the longer operative time was due to the use of open surgery in DS at that time. Furthermore, the overall risk of early postoperative complications in the present cohort of patients with elevated BMI is almost doubled (14\% vs $7 \%$ ) compared with all patients having bariatric surgery in Sweden (mean BMI 41) [27].

\section{Incidence Rate of Marginal Ulcer}

The present incidence rate of MU was just above $1 \%$, i.e., only one out of 100 operated patients will suffer an MU annually. 
Table 2 Symptoms leading to endoscopy and diagnosis of marginal ulcer, as well as any additional diagnoses, in the 23 patients having had gastroscopy

\begin{tabular}{|c|c|c|c|c|c|}
\hline Patient & Abdominal pain & GI bleeding/anemia & Dysphagia & Additional diagnoses & Procedure \\
\hline 1 & Yes & & & & DS \\
\hline 2 & Yes & & & & DS \\
\hline 3 & & Bleeding & & & DS \\
\hline 4 & & Bleeding & & & DS \\
\hline 5 & Yes & Anemia & Yes & Gastritis & DS \\
\hline 6 & & Anemia & & & DS \\
\hline 7 & & Bleeding & & & DS \\
\hline 8 & & Bleeding & & & DS \\
\hline 9 & Yes & & Yes & & RYGBP \\
\hline 10 & Yes & & & & RYGBP \\
\hline 11 & & Bleeding & & & RYGBP \\
\hline 12 & & & Yes & & RYGBP \\
\hline 13 & & & Yes & GJ stenosis & RYGBP \\
\hline 14 & Yes & & Yes & GJ stenosis & RYGBP \\
\hline 15 & Yes & & & & RYGBP \\
\hline 16 & & Anemia & & & RYGBP \\
\hline 17 & & Bleeding & & & RYGBP \\
\hline 18 & & & Yes & GJ stenosis & RYGBP \\
\hline 19 & Yes & Bleeding & & & RYGBP \\
\hline 20 & & Bleeding & & & RYGBP \\
\hline 21 & Yes & & & & RYGBP \\
\hline 22 & & Bleeding & Yes & GJ stenosis & RYGBP \\
\hline 23 & Yes & Bleeding & & & RYGBP \\
\hline
\end{tabular}

As mentioned earlier, Hess et al. described an incidence of $0.3 \%$ under a follow-up period of over 10 years in DS, whereas Pata and Scopinaro demonstrated a higher incidence of MU in classic biliopancreatic diversion, $2 \%$ and $3.2 \%$, respectively $[28,29]$. The difference could perhaps depend on the larger gastric remnant in biliopancreatic diversion, resulting in a higher amount of gastric acid passing through the upper anastomosis. This theory is supported by an earlier study by our group on RYGBP, in which we were able to show that the time with $\mathrm{pH}<4$ in the gastrojejunostomy was longer in patients with MU (69\%) compared with in non-MUs $(20 \%, p<$ $0.01)$ [14]. Interestingly, these absolute numbers are very similar to the time with $\mathrm{pH}<4$ in non-symptomatic DS patients, $59 \%$ in the acid-producing sleeve and $14 \%$ in the buffered duodenal bulb, as mentioned in the introduction. It would therefore be of great interest to measure the acidity in the
Table 3 Univariate and multivariate analyses of clinical important factors associative to developing a marginal ulcer

\begin{tabular}{|c|c|c|c|c|c|c|}
\hline & \multicolumn{3}{|c|}{ Univariate analysis } & \multicolumn{3}{|c|}{ Multivariate analysis* } \\
\hline & Odds ratio & $95 \% \mathrm{CI}$ & $\mathrm{P}$ value & Odds ratio & $95 \% \mathrm{CI}$ & $p$ value \\
\hline Gender & 1.31 & $0.65-2.65$ & 0.442 & 0.63 & $0.20-1.97$ & 0.433 \\
\hline Age at surgery & 0.99 & $0.96-1.02$ & 0.602 & 0.96 & $0.91-1.01$ & 0.185 \\
\hline BMI & 0.97 & $0.91-1.03$ & 0.405 & 0.89 & $0.75-1.07$ & 0.241 \\
\hline Any comorbidity & 0.64 & $0.33-1.23$ & 0.189 & 0.55 & $0.16-1.87$ & 0.346 \\
\hline Postop complication & 0.76 & $0.32-2.29$ & 0.767 & 1.52 & $0.34-6.76$ & 0.578 \\
\hline Surgical method & 0.86 & $0.45-1.67$ & 0.671 & 2.48 & $0.70-8.77$ & 0.158 \\
\hline BMI loss at 2 years & 1.02 & $0.94-1.10$ & 0.562 & 1.03 & $0.93-1.14$ & 0.566 \\
\hline Smoking & 1.79 & $0.44-3.15$ & 0.743 & 0.39 & $0.04-3.47$ & 0.401 \\
\hline Ongoing PPI & 9.70 & $4.68-20.10$ & $<0.001$ & 11.27 & $3.65-34.78$ & $<0.001$ \\
\hline
\end{tabular}

*Nagelkerke $R$-square $0.248 ; 203$ cases included 
present patients with MU. In the background population, the lifetime prevalence of a peptic ulcer is estimated to be $5-10 \%$ with an incidence of $0.1-0.3 \%$ per year [30,31]. Thus, the risk for having an ulcer is ten-fold in patients with a history of bariatric surgery. The majority of MU presented within the first 2 years, which is in line with several other studies [17, 19, 32]. Furthermore, none of our patients suffered a repeated MU nor a perforated MU, which otherwise is reported in 0.25 to $1 \%$ of cases [33].

\section{Difference Between our Survey and SOReg-Data}

Compared with SOReg-data, the present survey had a trend towards higher overall incidence rate for MU (1.4\% vs $0.9 \%$, $p=0.084)$ as well as MU in RYGBPs $(1.5 \%$ vs $0.7 \%, p=$ 0.067 ). This is probably due to more detailed data obtained by our questionnaire and the fact that SOReg-registrations are made in routine clinical care. Moreover, the diminishing follow-up rate of our patients in SOReg (38\% and $21 \%$ at 2 and 5 years, respectively) reduces the possibility of having a registered MU.

\section{Ongoing Treatment with PPI}

PPI treatment leads to complete resolution in all MUs. Persisting PPI treatment was seen in almost three quarters of our former MU patients, thus highly associated to MU (OR 11.2 [3.6-34.7], $p<0.001)$. This high number indicates either an increased vulnerability to acid-related conditions or a concern among patients and physicians of having a repeated ulcer. Furthermore, a quarter of the patients in the present cohort had ongoing PPI treatment, which is higher than in an earlier national study by our group on gastrointestinal symptoms 5 years after bariatric surgery. In that study, we were able to verify the good anti-acidic effect in RYGBP as the use of PPI was significantly reduced postoperatively, from 9.9 to $6.4 \%$ of the patients [34]. However, in general practice ( $n=40,000$ patients), it was found that a surprisingly large proportion, $14.7 \%$, were PPI users and the author concluded that a substantial number of individuals did not meet the correct indications for prescription [35]. Finally, in minimizing the risk for MU development in bariatric surgery, there is an increasing trend to administer PPI as a postoperative prophylaxis. However, neither a clear consensus on the duration of such treatment ( 30 or 90 days) nor solid evidence of its effect in the long term exists [20, 22, 36, 37].

\section{Limitations}

The well-defined cohort and thorough review of patients' charts is a strength of the present study, thus adding to the limited number of studies on MU in DS. Although the number of patients $(n=472)$ is a limitation, the present work provides a more accurate incidence rate of MU than in former registry studies. The response rate was not optimal (64\%); however, in a sensitivity analysis, responders only differed from the remaining patients by being older (41 vs 34 years) and more often having comorbidities ( $65 \%$ vs $55 \%, p<0.05$ for both). Moreover, we lack knowledge of peptic ulcer history as well as preoperative PPI use and smoking habits at time of MU diagnosis, three factors demonstrated to be of importance in gastric bypass patients [38]. Finally, the low number of endoscopically verified MU is a limitation; however, in all patients, a formal diagnosis of MU was set in medical charts.

In conclusion, the incidence rate of marginal ulcers after duodenal switch and gastric bypass was low, around $1 \%$, and did not differ between the two procedures. We believe that this is reassuring for future bariatric surgery candidates as well as bariatric surgeons.

Acknowledgments We would like to acknowledge Mr. Hans Högberg for his excellent contribution to statistical analyses.

Funding Information Open access funding provided by Uppsala University.

\section{Compliance with Ethical Standards}

Conflict of Interest The authors declare that they have no conflict of interest.

Ethical Approval The study was approved by the regional ethical committee, Uppsala, Sweden (Dnr 2018/088). All procedures performed in studies involving human participants were in accordance with the ethical standards of the institutional and/or national research committee and with the 1964 Helsinki declaration and its later amendments or comparable ethical standards.

Informed Consent Written informed consent was obtained from all individual participants included in the study.

Open Access This article is licensed under a Creative Commons Attribution 4.0 International License, which permits use, sharing, adaptation, distribution and reproduction in any medium or format, as long as you give appropriate credit to the original author(s) and the source, provide a link to the Creative Commons licence, and indicate if changes were made. The images or other third party material in this article are included in the article's Creative Commons licence, unless indicated otherwise in a credit line to the material. If material is not included in the article's Creative Commons licence and your intended use is not permitted by statutory regulation or exceeds the permitted use, you will need to obtain permission directly from the copyright holder. To view a copy of this licence, visit http://creativecommons.org/licenses/by/4.0/.

\section{References}

1. Nguyen NT, Magno CP, Lane KT, et al. Association of hypertension, diabetes, dyslipidemia, and metabolic syndrome with obesity: findings from the National Health and Nutrition Examination Survey, 1999 to 2004. JACS. 2008;207(6):928-34. 
2. Health effects of overweight and obesity in 195 countries over 25 years. N Engl J Med. 2017;377(1):13-27.

3. Sjostrom L, Narbro K, Sjostrom CD, et al. Effects of bariatric surgery on mortality in Swedish obese subjects. N Engl J Med. 2007:357(8):741-52.

4. Buchwald H, Avidor Y, Braunwald E, et al. Bariatric surgery: a systematic review and meta-analysis. JAMA. 2004;292(14):172437.

5. Angrisani L, Santonicola A, Iovino P, et al. Bariatric surgery and endoluminal procedures: IFSO Worldwide Survey 2014. Obes Surg. 2017;27(9):2279-89.

6. Topart P, Becouarn G, Ritz P. Weight loss is more sustained after biliopancreatic diversion with duodenal switch than Roux-en-Y gastric bypass in superobese patients. Surg Obes Relat Dis. 2013;9(4):526-30.

7. Hess DS, Hess DW, Oakley RS. The biliopancreatic diversion with the duodenal switch: results beyond 10 years. Obes Surg. 2005;15(3):408-16.

8. Skogar ML, Sundbom M. Early complications, long-term adverse events, and quality of life after duodenal switch and gastric bypass in a matched national cohort. Surg Obes Relat Dis. 2020;

9. Biertho L, Lebel S, Marceau S, et al. Perioperative complications in a consecutive series of 1000 duodenal switches. Surg Obes Relat Dis. 2013;9(1):63-8.

10. Bolckmans R, Himpens J. Long-term (>10 Yrs) outcome of the laparoscopic biliopancreatic diversion with duodenal switch. Ann Surg. 2016;264(6):1029-37.

11. Sapala JA, Wood MH, Sapala MA, et al. Marginal ulcer after gastric bypass: a prospective 3-year study of 173 patients. Obes Surg Okt. 1998;8(5):505-16.

12. Pyke O, Yang J, Cohn T, et al. Marginal ulcer continues to be a major source of morbidity over time following gastric bypass. Surg Endosc Okt. 2019;33(10):3451-6.

13. Bhayani NH, Oyetunji TA, Chang DC, et al. Predictors of marginal ulcers after laparoscopic Roux-en-Y gastric bypass. J Surg Res. 2012;177(2):224-7.

14. Hedberg J, Hedenstrom $\mathrm{H}$, Nilsson S, et al. Role of gastric acid in stomal ulcer after gastric bypass. Obes Surg. 2005;15(10):1375-8.

15. Coblijn UK, Lagarde SM, de Castro SMM, et al. Symptomatic marginal ulcer disease after Roux-en-Y gastric bypass: incidence, risk factors and management. Obes Surg. 2015;25(5):805-11.

16. Spaniolas K, Yang J, Crowley S, et al. Association of long-term anastomotic ulceration after Roux-en-Y gastric bypass with tobacco smoking. JAMA Surg. 2018;153(9):862-4.

17. Azagury D, Abu Dayyeh B, Greenwalt I, et al. Marginal ulceration after Roux-en-Y gastric bypass surgery: characteristics, risk factors, treatment, and outcomes. Endoscopy. 2011;43(11):950-4.

18. El-Hayek K, Timratana P, Shimizu H, et al. Marginal ulcer after Roux-en-Y gastric bypass: what have we really learned? Surg Endosc. 2012;26(10):2789-96.

19. Palermo M, Acquafresca PA, Rogula T, et al. Late surgical complications after gastric by-pass: a literature review. Arq Bras Cir Dig. 2015;28(2):139-43.

20. Ying VWC, Kim SHH, Khan KJ, et al. Prophylactic PPI help reduce marginal ulcers after gastric bypass surgery: a systematic review and meta-analysis of cohort studies. Surg Endosc. May 2015;29(5):1018-23.
21. Dallal RM, Bailey LA. Ulcer disease after gastric bypass surgery. Surg Obes Relat Dis. 2006;2(4):455-9.

22. Gumbs AA, Duffy AJ, Bell RL. Incidence and management of marginal ulceration after laparoscopic Roux-Y gastric bypass. Surg Obes Relat Dis. Aug 2006;2(4):460-3.

23. MacLean LD, Rhode BM, Nohr C, et al. Stomal ulcer after gastric bypass. J Am Coll Surg. 1997;185(1):1-7.

24. Clapp B, Hahn J, Dodoo C, et al. Evaluation of the rate of marginal ulcer formation after bariatric surgery using the MBSAQIP database. Surg Endosc. Jun 2019;33(6):1890-7.

25. Bekhali Z, Hedberg J, Hedenstrom $\mathrm{H}$, et al. Large buffering effect of the duodenal bulb in duodenal switch: a wireless $\mathrm{pH}$-metric study. Obes Surg. 2017;27(7):1867-71.

26. Tao W, Holmberg D, Naslund E, et al. Validation of obesity surgery data in the Swedish National Patient Registry and Scandinavian Obesity Registry (SOReg). Obes Surg. 2016;26(8):1750-6.

27. SOReg Årsrapport 2018 Del-1 [Internet]. 2019. Available at: https://www.ucr.uu.se/soreg/component/edocman/arsrapport2018-del-1

28. Pata G, Crea N, Di Betta E, et al. Biliopancreatic diversion with transient gastroplasty and duodenal switch: long-term results of a multicentric study. Surgery. 2013;153(3):413-22.

29. Scopinaro N, Adami GF, Marinari GM, et al. Biliopancreatic diversion. World J Surg. 1998;22(9):936-46.

30. Lanas A, Chan FKL. Peptic ulcer disease. Lancet. 2017;390(10094):613-24.

31. Lin KJ, Garcia Rodriguez LA, Hernandez-Diaz S. Systematic review of peptic ulcer disease incidence rates: do studies without validation provide reliable estimates? Pharmacoepidemiol Drug Saf. 2011;20(7):718-28.

32. Moon RC, Teixeira AF, Goldbach M, et al. Management and treatment outcomes of marginal ulcers after Roux-en-Y gastric bypass at a single high volume bariatric center. Surg Obes Relat Dis. 2014;10(2):229-34.

33. Kalaiselvan R, Exarchos G, Hamza N, et al. Incidence of perforated gastrojejunal anastomotic ulcers after laparoscopic gastric bypass for morbid obesity and role of laparoscopy in their management. Surg Obes Relat Dis. 2012;8(4):423-8.

34. Elias K, Hedberg J, Sundbom M. Prevalence and impact of acidrelated symptoms and diarrhea in patients undergoing Roux-en-Y gastric bypass, sleeve gastrectomy, and biliopancreatic diversion with duodenal switch. Surg Obes Relat Dis. 2019;

35. Tosetti C, Nanni I. Use of proton pump inhibitors in general practice. World J Gastrointest Pharmacol Ther. 2017;8(3):180-5.

36. Kang X, Zurita-Macias L, Hong D, et al. A comparison of 30-day versus 90-day proton pump inhibitor therapy in prevention of marginal ulcers after laparoscopic Roux-en-Y gastric bypass. Surg Obes Relat Dis. 2016;12(5):1003-7.

37. Wennerlund J, Gunnarsson U, Strigard K, et al. Acid-related complications after laparoscopic Roux-en-Y gastric bypass: risk factors and impact of proton pump inhibitors. Surg Obes Relat Dis. 2020;

38. Sverden E, Mattsson F, Sonden A, et al. Risk factors for marginal ulcer after gastric bypass surgery for obesity: a population-based cohort study. Ann Surg. 2016;263(4):733-7.

Publisher's Note Springer Nature remains neutral with regard to jurisdictional claims in published maps and institutional affiliations. 